\title{
Weakly collision-free paths for continuous humanoid footstep planning
}

\author{
Nicolas Perrin and Olivier Stasse and Florent Lamiraux and Eiichi Yoshida
}

\begin{abstract}
In this paper we demonstrate an original equivalence between footstep planning problems, where discrete sequences of steps are searched for, and the more classical problem of motion planning for a $2 \mathrm{D}$ rigid shape, where a continuous collision-free path has to be found. This equivalence enables a lot of classical motion planning techniques (such as PRM, RRT, etc.) to be applied almost effortlessly to the specific problem of footstep planning for a humanoid robot.
\end{abstract}

\section{INTRODUCTION}

Humanoid robots move continuously but contacts with the ground change in a discrete manner. This hybrid behavior provides humanoid robot with the unique ability to cross obstacles by stepping over them, but it also makes the motion planning problem very different from the classical case. As a result, the classical methods don't apply, and specific methods had to be designed. A lot of approaches for footstep planning are based on the $A^{*}$ search (see [12], [6], [7], [9]), which doesn't explore the workspace as fast as samplingbased motion planning techniques such as rapidly-exploring random trees (RRTs) for example (see [15]). The same is true for methods using discrete-time nonholonomic systems ([3]) or optimization problems ([16]) as they can be trapped in local minima. To enhance performance a good approach is to use tiered planning strategies ([8]), but it can be hard to know exactly what is lost when using several layers of motion planning: indeed, a high level planner might miss existing solutions that would have been found by the lower level planner. It is the case for example when a bounding box is used for humanoid motion planning (see [18]): since a collision-free path must be found for the bounding box, all the obstacles, even the smallest ones, must be circumvented, and as a consequence the robot cannot use its stepping over ability. Other approaches attempt to mimic human behavior by combining several strategies that correspond to different tasks ([4]), but when the list of strategies is long it can become quite difficult to predict and analyze the behavior and soundness of the global algorithm.

In this paper, we consider the problem of 2D footstep planning, with only 2D obstacles (i.e. holes in the ground), and show that for a particular instance of stepping capabilities (a rather natural one), there is an equivalence between finding

This work was supported by a grant from the RBLINK Project, Contract ANR-08-JCJC-0075-01.

N. Perrin is with CNRS/LAAS, Université de Toulouse UPS, INSA, INP, ISAE, F-31077 Toulouse, France and CNRS-AIST Joint Robotics Laboratory, UMI3218/CRT, Tsukuba, Japan n . perrineaist.go. jp

O. Stasse and E. Yoshida are with CNRS-AIST Joint Robotics Laboratory, UMI3218/CRT, Tsukuba, Japan. \{olivier.stasse, e.yoshida\}eaist.go.jp

F. Lamiraux is with CNRS/LAAS, Université de Toulouse UPS, INSA, INP, ISAE, F-31077 Toulouse, France. florentelaas. fr

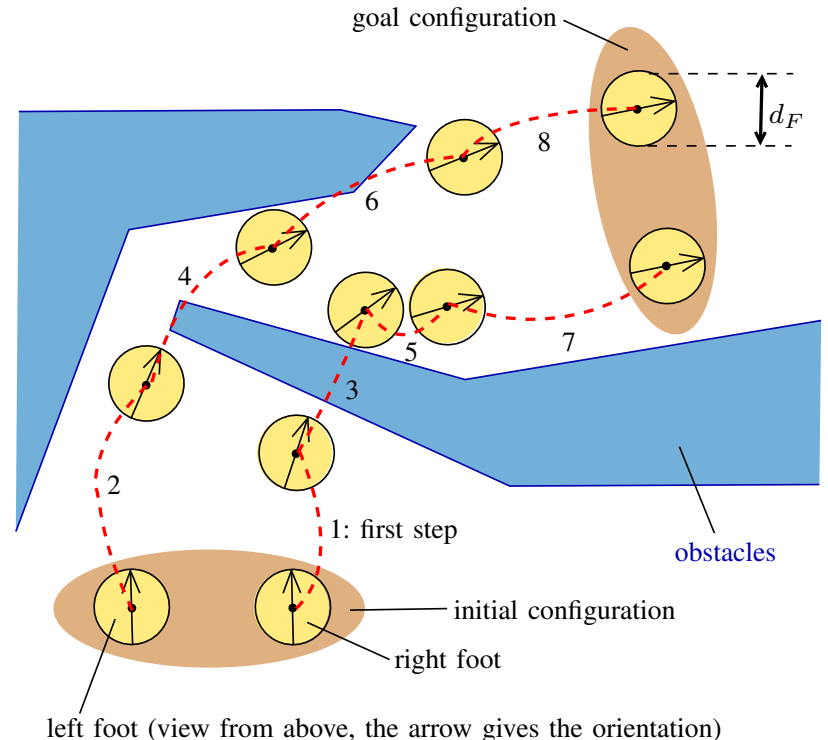

Fig. 1. An example of 2D footstep planning problem.

a discrete sequence of steps towards a goal and solving the classical motion planning problem for a simple 2D shape that can translate and rotate. Hence classical methods such as RRT or probabilistic roadmaps (PRM, see [11]) can be very easily applied to solve the footstep planning problem.

First, in section II, we define the stepping capabilities of the robot and state the problem and the main theorem. Then, in section III and IV we prove the two implications of the equivalence. In section $\mathrm{V}$ and VI, we mention the potential applications of our result, and show that it can be generalized to robots with different stepping capabilities. Section VII is the conclusion.

\section{PROBLEM STATEMENT}

Let us first describe the stepping capabilites of the robot we consider. As shown on Fig. 1, its feet have the shape of a circle of diameter $d_{F}$.

As mentioned in the introduction we consider the followingg 2D motion planning problem: the robot must find a discrete sequence of footprints that leads to a goal location without intersecting any obstacle.

When on the ground, the configuration of a foot is defined by three parameters: two for its position and one for its orientation. A stance is defined by two feet configurations (one for the left foot, one for the right foot). Now we start to define the particular stepping capabilites that are needed for the theorem we prove below: for a stance to be 


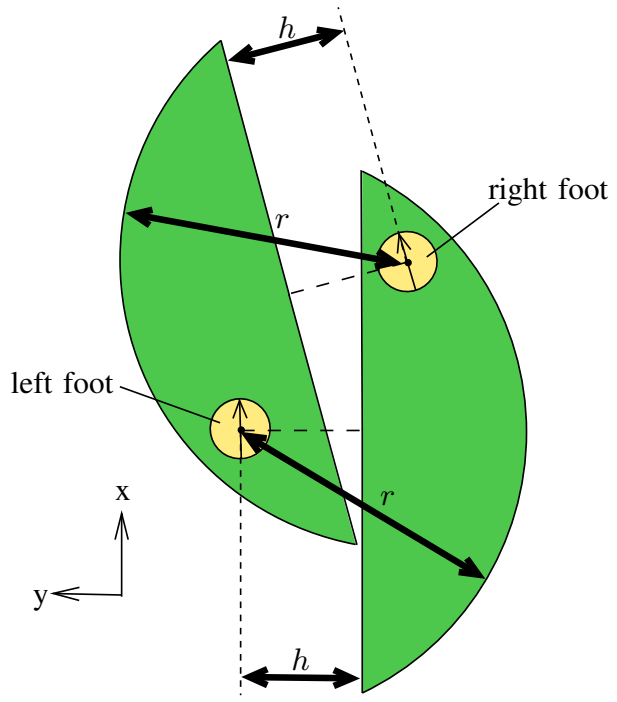

Fig. 2. A part of our hypothesis is that for a stance to be acceptable, a necessary condition is that each foot must be inside a region defined by the position and orientation of the other foot.

acceptable, both feet must be inside a portion of disk whose configuration is fixed relatively to the other foot (see Fig. 2). More formally, if we denote by $(x, y, \theta)$ the configuration of the left foot, then the set of acceptable positions for the right foot is the set $\mathcal{E}_{\mathcal{R}}(x, y, \theta) \subset \mathbb{R}^{2}$ of positions $\left(x^{\prime}, y^{\prime}\right)$ such that:

1) $\sin \theta \cdot\left(x^{\prime}-x\right)-\cos \theta \cdot\left(y^{\prime}-y\right) \geq h$

2) $\sqrt{\left(x^{\prime}-x\right)^{2}+\left(y^{\prime}-y\right)^{2}} \leq r$

Similarly, if we denote by $(x, y, \theta)$ the configuration of the right foot, then the set $\mathcal{E}_{\mathcal{L}}(x, y, \theta)$ of acceptable positions for the left foot is the set of positions $\left(x^{\prime}, y^{\prime}\right)$ such that:

1) $\sin \theta \cdot\left(x^{\prime}-x\right)+\cos \theta \cdot\left(y^{\prime}-y\right) \geq h$

2) $\sqrt{\left(x^{\prime}-x\right)^{2}+\left(y^{\prime}-y\right)^{2}} \leq r$

So, a necessary condition for a stance $\left((x, y, \theta),\left(x^{\prime}, y^{\prime}, \theta^{\prime}\right)\right)$ to be acceptable is that:

$$
\left(x^{\prime}, y^{\prime}\right) \in \mathcal{E}_{\mathcal{R}}(x, y, \theta) \text { and }(x, y) \in \mathcal{E}_{\mathcal{L}}\left(x^{\prime}, y^{\prime}, \theta^{\prime}\right)
$$

This constraint defines also implicit restrictions on the orientations, but additional restrictions can be imposed as well, and it will not change the stepping capabilities, as long as the following property is verified:

Definition 2.1: A constraint $C\left((x, y, \theta),\left(x^{\prime}, y^{\prime}, \theta^{\prime}\right)\right)$ on the stances is said to verify the $\mu$-property if there exists $\mu>0$ such that $\left|\theta^{\prime}-\theta\right|<\mu$ automatically implies that the constraint is satisfied.

The fact that additional constraints verifying the $\mu$-property do not change the stepping capabilities is surprising: it means that even with very restrictive constraints on the relative orientations of the feet, the stepping capabilities are not reduced.

But our stepping capabilities are not defined only by the constraints on the set of acceptable stances: an additional constraint that reduces the maximum length of feasible steps is imposed.

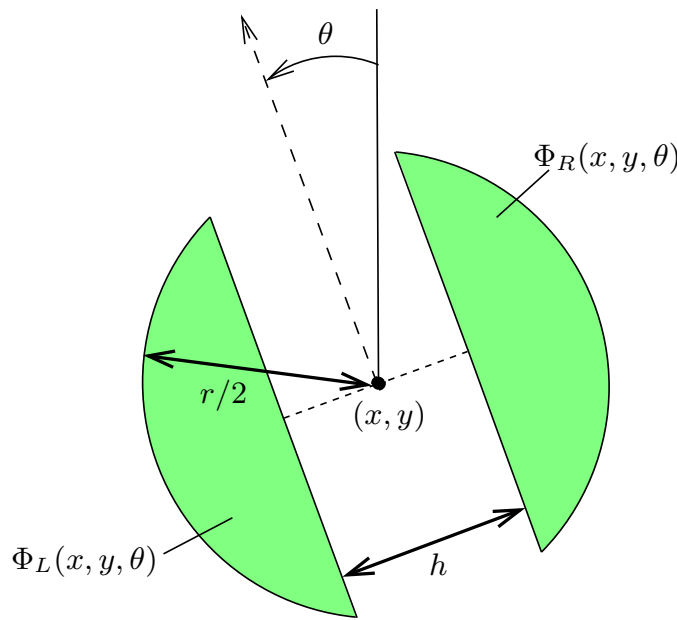

Fig. 3. The 2D object $\Phi$.

For this purpose we introduce the crucial 2D object shown on Fig. 3: it is composed of two symmetric portions of disk, and can be obtained by extruding an open stripe of width $h$ from a disk of radius $r / 2$ (where $r$ and $h$ are the same as the ones used to define the first constraints on the acceptable stances). Its configuration in the plane is defined by three parameters $x, y$ and $\theta$, where $(x, y)$ denote the position of its center and $\theta$ its orientation. We call $\Phi$ this object, and when in configuration $(x, y, \theta)$, we denote by $\Phi(x, y, \theta)$ the set of points it contains. $\Phi$ being divided into two parts, its orientation naturally defines a left and a right part. In configuration $(x, y, \theta)$, we denote respectively by $\Phi_{L}(x, y, \theta)$ and $\Phi_{R}(x, y, \theta)$ the sets of points contained in the left and right part of $\Phi$.

A step of the robot can be described by three triples: the configuration of the support foot, and the initial and final configurations of the swing foot. For example, if the left foot is the support foot, we can call $\left(x_{L}, y_{L}, \theta_{L}\right),\left(x_{R}^{i}, y_{R}^{i}, \theta_{R}^{i}\right)$ and $\left(x_{R}^{f}, y_{R}^{f}, \theta_{R}^{f}\right)$ these three configurations.

Definition 2.2: A step defined by such configurations is said feasible if and only if the following constraints are verified:

1) $\left(x_{R}^{i}, y_{R}^{i}\right) \in \mathcal{E}_{\mathcal{R}}\left(x_{L}, y_{L}, \theta_{L}\right)$

2) $\left(x_{L}, y_{L}\right) \in \mathcal{E}_{\mathcal{L}}\left(x_{R}^{i}, y_{R}^{i}, \theta_{R}^{i}\right)$

3) $\left(x_{R}^{f}, y_{R}^{f}\right) \in \mathcal{E}_{\mathcal{R}}\left(x_{L}, y_{L}, \theta_{L}\right)$

4) $\left(x_{L}, y_{L}\right) \in \mathcal{E}_{\mathcal{L}}\left(x_{R}^{f}, y_{R}^{f}, \theta_{R}^{f}\right)$

5) Possibly a finite number of additional constraints on the stances, all verifying the $\mu$-property (Definition 2.1).

6) There exists a configuration $(x, y, \theta)$ such that $\left(x_{L}, y_{L}\right),\left(x_{R}^{i}, y_{R}^{i}\right)$ and $\left(x_{R}^{f}, y_{R}^{f}\right)$ are all contained in $\Phi(x, y, \theta)$.

These constraints (and, of course, the symmetric constraints with the right support foot) completely describe the stepping capabilities of the robot. Fig. 4 shows a few feasible and unfeasible steps.

Now, we formulate the motion planning problem and state the main theorem of this paper. The obstacles are defined 


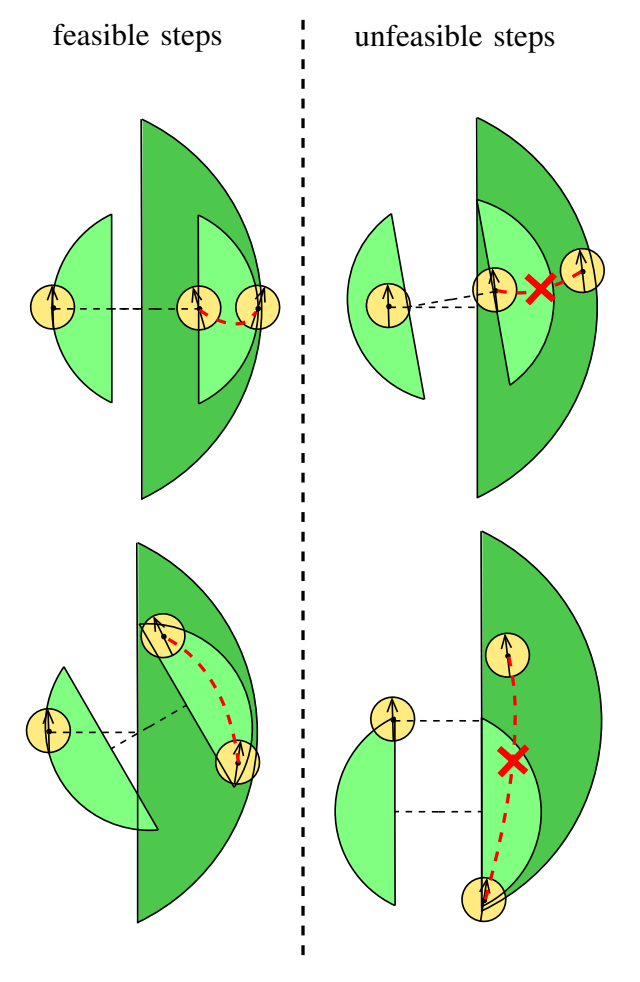

Fig. 4. Feasible and unfeasible steps.

by a finite number of closed sets of points in the plane. The robot starts with both feet on the ground, the left and right foot being respectively in configuration $\left(x_{L}^{0}, y_{L}^{0}, \theta_{L}^{0}\right)=$ $c_{L}^{0}$ and $\left(x_{R}^{0}, y_{R}^{0}, \theta_{R}^{0}\right)=c_{R}^{0}$, which are supposed collisionfree (i.e. the disks of radius $\frac{d_{F}}{2}$ and centers $\left(x_{L}^{0}, y_{L}^{0}\right)$ and $\left(x_{R}^{0}, y_{R}^{0}\right)$ don't intersect with any obstacle, even on their borders). A collision-free goal configuration is given for both feet $\left(\left(x_{L}^{G}, y_{L}^{G}, \theta_{L}^{G}\right)=c_{L}^{G}\right.$ and $\left.\left(x_{R}^{G}, y_{R}^{G}, \theta_{R}^{G}\right)=c_{R}^{G}\right)$, and the objective is to find a finite sequence of feasible steps that goes from $\left(c_{L}^{0}, c_{R}^{0}\right)$ to $\left(c_{L}^{G}, c_{R}^{G}\right)$ while avoiding all the obstacles. An example instance of this problem is shown on Fig. 1.

Before stating the main theorem, we give a special definition of "collision-free" for the object $\Phi$ :

Definition 2.3: A configuration $\Phi(x, y, \theta)$ of $\Phi$ is said to be "weakly collision-free" if and only if there exist $\left(x_{l}, y_{l}\right) \in$ $\Phi_{L}(x, y, \theta)$ and $\left(x_{r}, y_{r}\right) \in \Phi_{R}(x, y, \theta)$ such that there is no intersection between the obstacles and the two disks of radius $\frac{d_{F}}{2}$ and centers $\left(x_{l}, y_{l}\right)$ and $\left(x_{l}, y_{l}\right)$.

Fig. 5 shows a "weakly collision-free" configuration, and a continuous "weakly collision-free path", i.e. a continuous path $\mathcal{S}:[0,1] \mapsto \mathbb{R}^{2} \times S O(2)$ such that $\forall t \in[0,1], \Phi(\mathcal{S}(t))$ is weakly collision-free.

We can now state our main result:

Theorem 2.1: There exists a collision-free sequence of feasible steps from $\left(\left(x_{L}^{0}, y_{L}^{0}, \theta_{L}^{0}\right),\left(x_{R}^{0}, y_{R}^{0}, \theta_{R}^{0}\right)\right)$ to the goal if and only if there exists a continuous weakly collision-free path $\mathcal{S}:[0,1] \mapsto \mathbb{R}^{2} \times S O(2)$ such that:

1) $\left(x_{L}^{0}, y_{L}^{0}\right) \in \Phi_{L}(\mathcal{S}(0))$ and $\left(x_{R}^{0}, y_{R}^{0}\right) \in \Phi_{R}(\mathcal{S}(0))$

2) $\left(x_{L}^{G}, y_{L}^{G}\right) \in \Phi_{L}(\mathcal{S}(1))$ and $\left(x_{R}^{G}, y_{R}^{G}\right) \in \Phi_{R}(\mathcal{S}(1))$

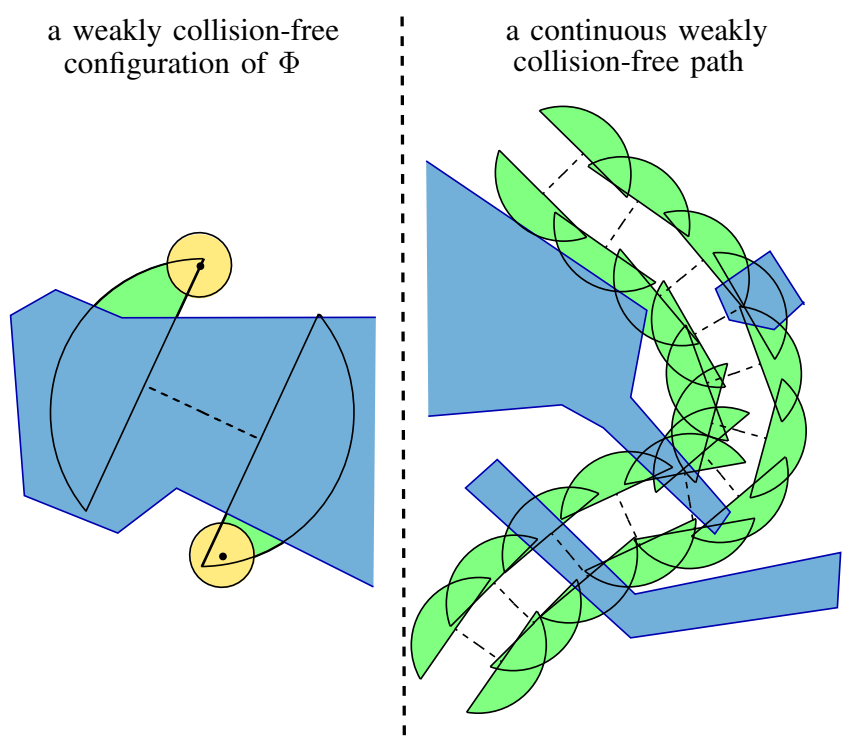

Fig. 5. Weak collision-freeness.

In the next two sections we demonstrate the two implications of this theorem.

\section{FROM A SOLUTION TO A WEAKLY COLLISION-FREE PATH}

We first show that the existence of a finite sequence of feasible steps from the initial configuration to the goal implies the existence of a continuous weakly collision-free path for $\Phi$.

Proof: Without loss of generality, we suppose that the first support foot is the right one, and that the last support foot is the left one. Let us denote by $\left(c_{R}^{0}, c_{L}^{0}, c_{L}^{1}\right),\left(c_{L}^{1}, c_{R}^{0}, c_{R}^{1}\right), \ldots,\left(c_{L}^{n}, c_{R}^{n-1}, c_{R}^{n}\right)=$ $\left(c_{L}^{G}, c_{R}^{n-1}, c_{R}^{G}\right)$ a sequence of feasible steps that goes to the goal while avoiding the obstacles (we recall that $\left(c_{R}^{a}, c_{L}^{a}, c_{L}^{b}\right)$ denotes the step from stance $\left(c_{L}^{a}, c_{R}^{a}\right)$ to $\left(c_{L}^{b}, c_{R}^{a}\right)$, and $\left(c_{L}^{a}, c_{R}^{a}, c_{R}^{b}\right)$ the step from stance $\left(c_{L}^{a}, c_{R}^{a}\right)$ to $\left.\left(c_{L}^{a}, c_{R}^{b}\right)\right)$.

For any step $\left(c_{R}^{i}, c_{L}^{i}, c_{L}^{i+1}\right)$, thanks to the constraint 6$)$ in definition 2.2, we know that there exists a configuration $\Phi\left(x_{2 i}, y_{2 i}, \theta_{2 i}\right)$ such that $\left(x_{R}^{i}, y_{R}^{i}\right) \in \Phi_{R}\left(x_{2 i}, y_{2 i} \theta_{2 i}\right)$ and $\left(x_{L}^{i}, y_{L}^{i}\right) \in \Phi_{L}\left(x_{2 i}, y_{2 i}, \theta_{2 i}\right)$ and $\left(x_{L}^{i+1}, y_{L}^{i+1}\right) \in$ $\Phi_{L}\left(x_{2 i}, y_{2 i}, \theta_{2 i}\right)$. Since the steps avoid the obstacles, this configuration is necessarily weakly collision-free. Similarly, for any step $\left(c_{L}^{i+1}, c_{R}^{i}, c_{R}^{i+1}\right)$ there exists a weakly collision-free configuration $\Phi\left(x_{2 i+1}, y_{2 i+1}, \theta_{2 i+1}\right)$ containing the three points in the corresponding parts of $\Phi$.

We show that there exists a continuous weakly collisionfree path $\mathcal{S}:[0,1] \mapsto \mathbb{R}^{2} \times S O(2)$ such that for all $k \in\{0, \ldots, 2 n-1\}, \mathcal{S}\left(\frac{k}{2 n-1}\right)=\left(x^{k}, y^{k}, \theta^{k}\right)$. To obtain this result it is enough to prove that $\forall k \in\{0, \ldots, 2 n-2\}$, there is a continuous weakly collision-free path $\mathcal{S}^{k}:\left[\frac{k}{2 n-1}, \frac{k+1}{2 n-1}\right] \mapsto$ $\mathbb{R}^{2} \times S O(2)$ such that $\mathcal{S}^{k}\left(\frac{k}{2 n-1}\right)=\left(x^{k}, y^{k}, \theta^{k}\right)$ and $\mathcal{S}^{k}\left(\frac{k+1}{2 n-1}\right)=\left(x^{k+1}, y^{k+1}, \theta^{k+1}\right)$. We do it only for $k=0$ (the same demonstration applies for any $k \in\{0, \ldots, 2 n-$ $2\})$. In that case, there are 4 foot positions to consider: 


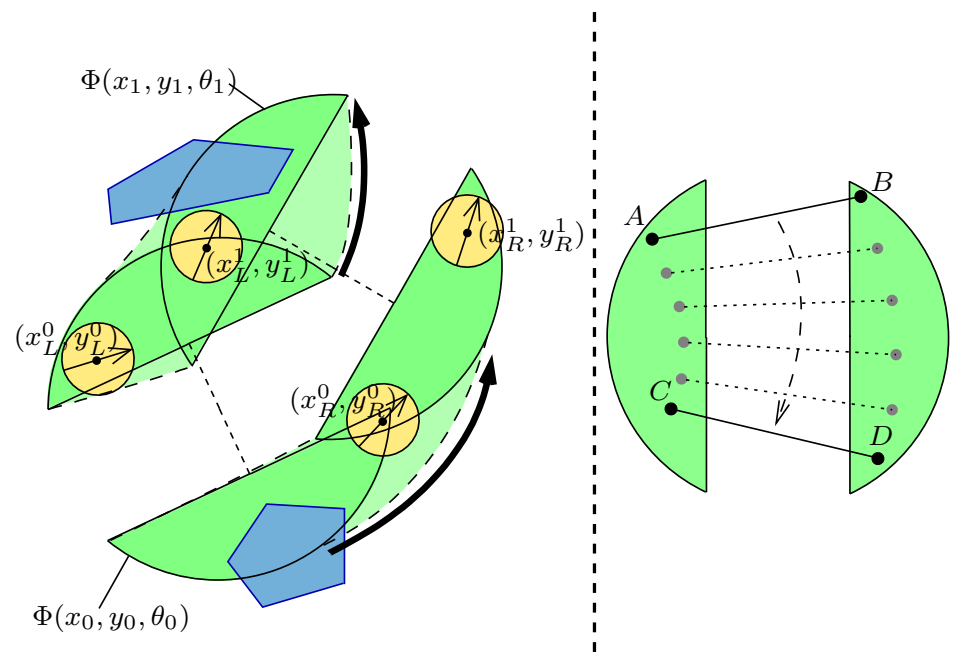

Fig. 6. On the left: a continuous weakly collision-free path from $\Phi\left(x_{0}, y_{0}, \theta_{0}\right)$ to $\Phi\left(x_{1}, y_{1}, \theta_{1}\right)$, keeping $\left(x_{L}^{1}, y_{L}^{1}\right)$ and $\left(x_{R}^{0}, y_{R}^{0}\right)$ in $\Phi$ at all time. On the right, a dual transformation: moving continuously the segment line $A B$ towards $C D$ while keeping its extremities inside $\Phi$.

$\left(x_{R}^{0}, y_{R}^{0}\right),\left(x_{L}^{0}, y_{L}^{0}\right),\left(x_{R}^{1}, y_{R}^{1}\right)$ and $\left(x_{L}^{1}, y_{L}^{1}\right) . \Phi\left(x_{0}, y_{0}, \theta_{0}\right)$ contains $\left(x_{R}^{0}, y_{R}^{0}\right),\left(x_{L}^{0}, y_{L}^{0}\right)$ and $\left(x_{L}^{1}, y_{L}^{1}\right)$ while $\Phi\left(x_{1}, y_{1}, \theta_{1}\right)$ contains $\left(x_{L}^{1}, y_{L}^{1}\right),\left(x_{R}^{0}, y_{R}^{0}\right)$ and $\left(x_{R}^{1}, y_{R}^{1}\right)$. Fig. 6 sums up the situation. The key point is that $\left(x_{R}^{0}, y_{R}^{0}\right)$ and $\left(x_{L}^{1}, y_{L}^{1}\right)$ are contained in both $\Phi\left(x_{0}, y_{0}, \theta_{0}\right)$ and $\Phi\left(x_{1}, y_{1}, \theta_{1}\right)$. Because of the particular shape of $\Phi$, it is possible to show that we can go continuously from $\Phi\left(x_{0}, y_{0}, \theta_{0}\right)$ to $\Phi\left(x_{1}, y_{1}, \theta_{1}\right)$ while keeping $\left(x_{R}^{0}, y_{R}^{0}\right)$ in $\Phi_{L}$ and $\left(x_{L}^{1}, y_{L}^{1}\right)$ in $\Phi_{R}$. We will not explain it in detail but this is a consequence of the following property: considering a fixed configuration of $\Phi$, for any couple of line segments of same length $A B$ and $C D$, such that $A, C \in \Phi_{L}$ and $B, D \in \Phi_{R}$, it is possible to continuously move $A B$ until it coincides with $C D$, without ever moving $A$ (resp. $B$ ) out of $\Phi_{L}$ (resp. $\Phi_{R}$ ). On Fig. 6 $A$ and $C$ would both correspond to $\left(x_{L}^{1}, y_{L}^{1}\right)$, and $B$ and $D$ would both correspond to $\left(x_{R}^{0}, y_{R}^{0}\right)$. There are other shapes than $\Phi$ verifying the same property, but $\Phi$ is one of the simplest.

The path resulting from the continuous displacement from $\Phi\left(x_{0}, y_{0}, \theta_{0}\right)$ to $\Phi\left(x_{1}, y_{1}, \theta_{1}\right)$ verifies the properties that we were looking for: since it always contains $\left(x_{R}^{0}, y_{R}^{0}\right)$ and $\left(x_{L}^{1}, y_{L}^{1}\right)$ it is clearly weakly collision-free. Connecting the paths obtained for $k=0,1, \ldots, 2 n-2$ gives us a continuous weakly collision-free path from $\left(x^{0}, y^{0}, \theta^{0}\right)$ to $\left(x^{2 n-1}, y^{2 n-1}, \theta^{2 n-1}\right)$, and that concludes the demonstration.

\section{FROM A WEAKLY COLLISION-FREE PATH TO A SOLUTION}

In this section, we prove the converse implication: if there is a continuous weakly collision-free path for $\Phi$, then there exists a finite sequence of feasible steps from the initial configuration to the goal. It might be noted that this property is a bit similar to the reduction property in [2], where it is proven that a continuous collision-free solution to the

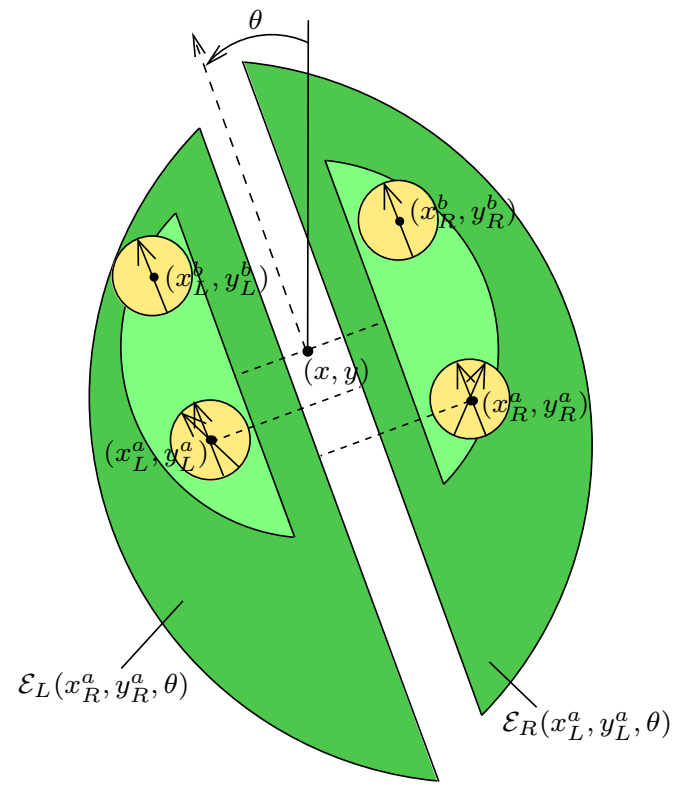

Fig. 7. There always exists a sequence of feasible steps from $\left(\left(x_{L}^{a}, y_{L}^{a}, \theta_{L}^{a}\right),\left(x_{R}^{a}, y_{R}^{a}, \theta_{R}^{a}\right)\right)$ to $\left(\left(x_{L}^{b}, y_{L}^{b}, \theta\right),\left(x_{R}^{b}, y_{R}^{b}, \theta\right)\right)$.

manipulation planning problem can always be converted into a finite sequence of manipulation tasks with fixed grasping configurations.

First, we prove the following lemma:

Lemma 4.1: Let $(x, y, \theta)$ be a configuration of $\Phi$, and $\left(x_{L}^{a}, y_{L}^{a}\right),\left(x_{L}^{b}, y_{L}^{b}\right)$ two points in $\Phi_{L}(x, y, \theta)$, and $\left(x_{R}^{a}, y_{R}^{a}\right)$, $\left(x_{R}^{b}, y_{R}^{b}\right)$ two points in $\Phi_{R}(x, y, \theta)$. We assume that these four points are at a distance greater than $\frac{d_{F}}{2}$ from the obstacles. Then from any acceptable stance based on $\left(x_{L}^{a}, y_{L}^{a}\right)$ and $\left(x_{R}^{a}, y_{R}^{a}\right)$ there exists a collision-free sequence of feasible steps to an acceptable stance based on $\left(x_{L}^{b}, y_{L}^{b}\right)$ and $\left(x_{R}^{b}, y_{R}^{b}\right)$.

Proof: Let us denote by $\left(x_{L}^{a}, y_{L}^{a}, \theta_{L}^{a}\right)$ and $\left(x_{R}^{a}, y_{R}^{a}, \theta_{R}^{a}\right)$ the two initial configurations of the feet. These two configurations form an acceptable stance, so we know that $\left(x_{R}^{a}, y_{R}^{a}\right) \in \mathcal{E}_{R}\left(x_{L}^{a}, y_{L}^{a}, \theta_{L}^{a}\right)$ and $\left(x_{L}^{a}, y_{L}^{a}\right) \in \mathcal{E}_{L}\left(x_{R}^{a}, y_{R}^{a}, \theta_{R}^{a}\right)$, with possible additional constraints verified. Because of the symmetry between $\mathcal{E}_{L}$ and $\mathcal{E}_{R}$, it is also possible to show that $\left(x_{L}^{a}, y_{L}^{a}\right) \in \mathcal{E}_{L}\left(x_{R}^{a}, y_{R}^{a}, \theta_{L}^{a}\right)$. Besides, any constraint verifying the $\mu$-property is satisfied by the stance $\left(\left(x_{L}^{a}, y_{L}^{a}, \theta_{L}^{a}\right),\left(x_{R}^{a}, y_{R}^{a}, \theta_{L}^{a}\right)\right)$. Since the point 6) of definition 2.2 is also verified, we deduce that the step $\left(\left(x_{L}^{a}, y_{L}^{a}, \theta_{L}^{a}\right),\left(x_{R}^{a}, y_{R}^{a}, \theta_{R}^{a}\right),\left(x_{R}^{a}, y_{R}^{a}, \theta_{L}^{a}\right)\right)$ is feasible. After this step both feet have the same orientation. Let us also consider the configurations $\left(x_{L}^{a}, y_{L}^{a}, \theta\right)$ and $\left(x_{R}^{a}, y_{R}^{a}, \theta\right)$ (we recall that $\theta$ is the orientation of $\Phi$ ). As shown on Fig. 7, we can easily verify that $\mathcal{E}_{R}\left(x_{L}^{a}, y_{L}^{a}, \theta\right) \supset$ $\Phi_{R}(x, y, \theta)$, and $\mathcal{E}_{L}\left(x_{R}^{a}, y_{R}^{a}, \theta\right) \supset \Phi_{L}(x, y, \theta)$. Therefore we have $\left(x_{R}^{a}, y_{R}^{a}\right) \in \mathcal{E}_{R}\left(x_{L}^{a}, y_{L}^{a}, \theta\right)$, and $\left(x_{L}^{a}, y_{L}^{a}\right) \in$ $\mathcal{E}_{L}\left(x_{R}^{a}, y_{R}^{a}, \theta\right)$. Thanks to this and to the $\mu$-property of the possible additional constraints on the stances, we can show that there exists a finite sequence of feasible steps that goes from stance $\left(\left(x_{R}^{a}, y_{R}^{a}, \theta_{R}^{a}\right),\left(x_{R}^{a}, y_{R}^{a}, \theta_{L}^{a}\right)\right)$ to stance $\left(\left(x_{R}^{a}, y_{R}^{a}, \theta\right),\left(x_{R}^{a}, y_{R}^{a}, \theta\right)\right)$. 
Once that the left and right foot are in respective configurations $\left(x_{L}^{a}, y_{L}^{a}, \theta\right)$ and $\left(x_{R}^{a}, y_{R}^{a}, \theta\right)$, it is easy to prove (see Fig. 7) that the steps leading first to $\left(\left(x_{L}^{b}, y_{L}^{b}, \theta\right),\left(x_{R}^{a}, y_{R}^{a}, \theta\right)\right)$ or $\left(\left(x_{L}^{a}, y_{L}^{a}, \theta\right),\left(x_{R}^{b}, y_{R}^{b}, \theta\right)\right)$, and then to $\left(\left(x_{L}^{b}, y_{L}^{b}, \theta\right),\left(x_{R}^{b}, y_{R}^{b}, \theta\right)\right)$, are also feasible. As a consequence, we obtained a sequence of collisionfree feasible steps from $\left(\left(x_{L}^{a}, y_{L}^{a}, \theta_{L}^{a}\right),\left(x_{R}^{a}, y_{R}^{a}, \theta_{R}^{a}\right)\right)$ to $\left(\left(x_{L}^{b}, y_{L}^{b}, \theta\right),\left(x_{R}^{b}, y_{R}^{b}, \theta\right)\right)$, which is an acceptable stance, and that concludes the proof of lemma 4.1.

We can now start the main demonstration.

Proof: Let $\mathcal{S}:[0,1] \mapsto \mathbb{R}^{2} \times S O(2)$ be a continuous weakly collision-free path of $\Phi$ towards the goal. For a configuration $(x, y, \theta)$ of $\Phi$, we denote by $d_{o b s}^{L}(x, y, \theta)$ the maximum distance between any point of $\Phi_{L}(x, y, \theta)$ and the obstacles, and by $d_{o b s}^{R}(x, y, \theta)$ the maximum distance between any point of $\Phi_{R}(x, y, \theta)$ and the obstacles. We also pose:

$$
d_{o b s}(x, y, \theta)=\min \left(d_{o b s}^{L}(x, y, \theta), d_{o b s}^{R}(x, y, \theta)\right),
$$

and:

$$
d_{\text {min }}(\mathcal{S})=\min _{t \in[0,1]}\left(d_{o b s}(\mathcal{S}(t))\right)
$$

Since $\mathcal{S}$ is weakly collision-free, and since both $\Phi$ and the obstacles are represented by closed set of points, we know that:

$$
d_{\min }(\mathcal{S})>\frac{d_{F}}{2}
$$

Besides, $\mathcal{S}$ is uniformly continuous on $[0,1]$, and therefore for any $\rho>0$ there exists $\epsilon>0$ such that for all $\left(t_{1}, t_{2}\right) \in[0,1]^{2},\left|t_{1}-t_{2}\right|<\epsilon \Rightarrow\left\|\mathcal{S}\left(t_{1}\right)-\mathcal{S}\left(t_{2}\right)\right\|_{\infty}<\rho$, where $\|(\Delta x, \Delta y, \Delta \theta)\|_{\infty}=\max (|\Delta x|,|\Delta y|,|\Delta \theta|$ ) (with $|\Delta \theta|$ being the smallest value among $\{|\Delta \theta+k 2 \pi|, k \in \mathbb{Z}\}$ ). It follows that for any $\eta>0$, there also exists $\epsilon>0$ such that $\left|t_{1}-t_{2}\right|<\epsilon$ implies that from $\mathcal{S}\left(t_{1}\right)$ to $\mathcal{S}\left(t_{2}\right)$ the points of $\Phi$ are moved by at most $\eta$. For a given $\eta>0$, let us denote by $\epsilon(\eta)$ a satisfying value of $\epsilon$, and let us denote by $N$ an integer such that $\frac{1}{N}<\epsilon(\eta)$.

We consider the configurations $\mathcal{S}\left(\frac{0}{N}\right), \mathcal{S}\left(\frac{1}{N}\right), \ldots, \mathcal{S}\left(\frac{N}{N}\right)$.

For $\eta$ small enough, we can show that for any $i \in$ $\{0, \ldots N-1\}$, every point in $\Phi_{L}\left(\mathcal{S}\left(\frac{i}{N}\right)\right) \cup \Phi_{L}\left(\mathcal{S}\left(\frac{i+1}{N}\right)\right)$ is at distance at most $\left(d_{\min }(\mathcal{S})-\frac{d_{F}}{2}\right) / 2$ from the nonempty intersection $\Phi_{L}\left(\mathcal{S}\left(\frac{i}{N}\right)\right) \cap \Phi_{L}\left(\mathcal{S}\left(\frac{i+1}{N}\right)\right)$, with the same property for $\Phi_{R}$. In that case, for any point $(x, y) \in$ $\Phi_{L}\left(\mathcal{S}\left(\frac{i}{N}\right)\right)$ at distance at least $d_{\min }(\mathcal{S})$ from the obstacles, there exists a point $\left(x^{\prime}, y^{\prime}\right) \in \Phi_{L}\left(\mathcal{S}\left(\frac{i}{N}\right)\right) \cap \Phi_{L}\left(\mathcal{S}\left(\frac{i+1}{N}\right)\right)$ at distance at least $d_{\min }(\mathcal{S})-\left(d_{\min }(\mathcal{S})-\frac{d_{F}}{2}\right) / 2>\frac{d_{F}}{2}$ from the obstacles. The result also applies for points in $\Phi_{R}^{2}$, and it follows that we can extract two sequence of points $\left(x_{L}^{1}, y_{L}^{1}\right), \ldots,\left(x_{L}^{N}, y_{L}^{N}\right)$ and $\left(x_{R}^{1}, y_{R}^{1}\right), \ldots,\left(x_{R}^{N}, y_{R}^{N}\right)$ that are all at distance greater than $\frac{d_{F}}{2}$ from the obstacles, and such that:

$\forall i \in\{1, \ldots, N\},\left(x_{L}^{i}, y_{L}^{i}\right) \in \Phi_{L}\left(\mathcal{S}\left(\frac{i-1}{N}\right)\right) \cap \Phi_{L}\left(\mathcal{S}\left(\frac{i}{N}\right)\right)$, and:

$\forall i \in\{1, \ldots, N\},\left(x_{R}^{i}, y_{R}^{i}\right) \in \Phi_{R}\left(\mathcal{S}\left(\frac{i-1}{N}\right)\right) \cap \Phi_{R}\left(\mathcal{S}\left(\frac{i}{N}\right)\right)$.

As an almost direct consequence of lemma 4.1, these points can be the support of a sequence of feasible steps

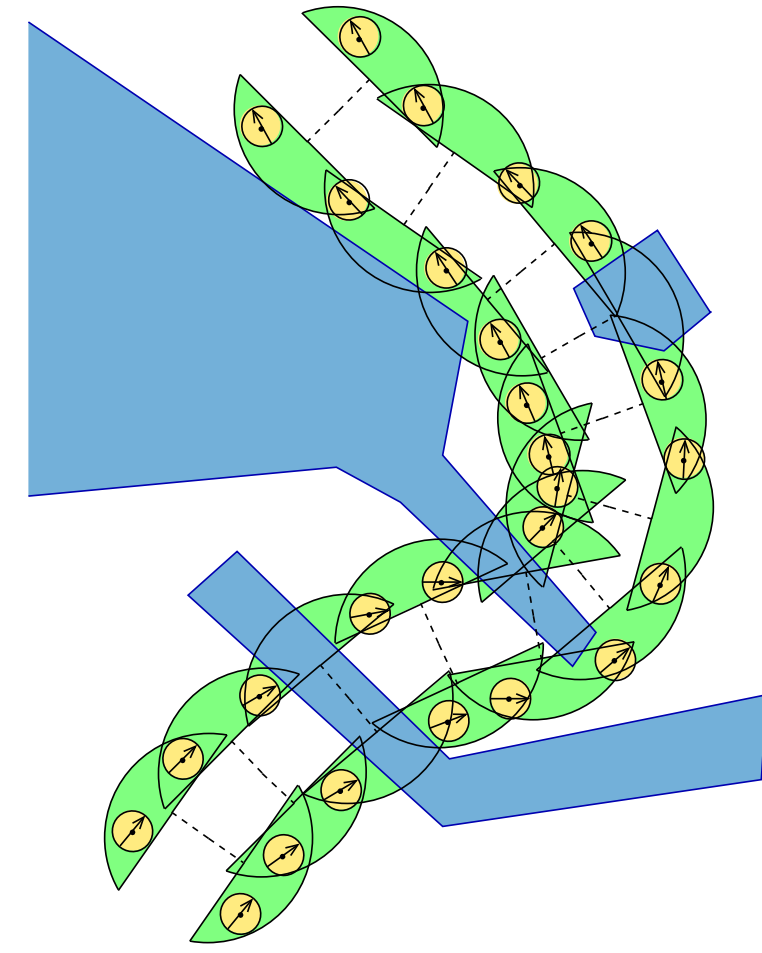

Fig. 8. From a continuous weakly collision-free path to a finite sequence of feasible collision-free steps.

going from the initial configuration to the goal. Fig. 8 illustrates the whole construction.

\section{APPLICATIONS}

For the same reason why the reduction property in [2] is useful for manipulation planning, our theorem is useful for footstep planning, as it turns an a priori specific problem of motion planning into an instance of a better-studied and more fundamental problem.

Indeed, the problem that consists in finding continuous collision-free paths (in $\mathbb{R}^{2} \times S O(2)$ ) for a robot represented as a rigid $2 \mathrm{D}$ shape that can translate and rotate, is the 2dimensional version of the most studied problem of motion planning: the classical piano's mover problem.

A great number of techniques have been designed to solve efficiently this problem, while fewer algorithms exist for footstep planning.

For example, as mentioned in the introduction, PRM and RRT are two widespread techniques of motion planning that cannot be directly applied to footstep planning. Thanks to theorem 2.1, it becomes the case: indeed, for a robot with the stepping capabilities described in section II, a sound way to solve the footstep planning problem is to first use PRM or RRT to find a continuous path for $\Phi$, and then convert it into a finite sequence of steps. Once a solution path $\mathcal{S}$ has been found, the conversion can be done very efficiently by a greedy algorithm which always tries to put the next stance of the robot in $\Phi(\mathcal{S}(t))$ with $t$ as large as possible.

During the execution of PRM or RRT, only one thing has to be changed: the collision checks must be replaced by 


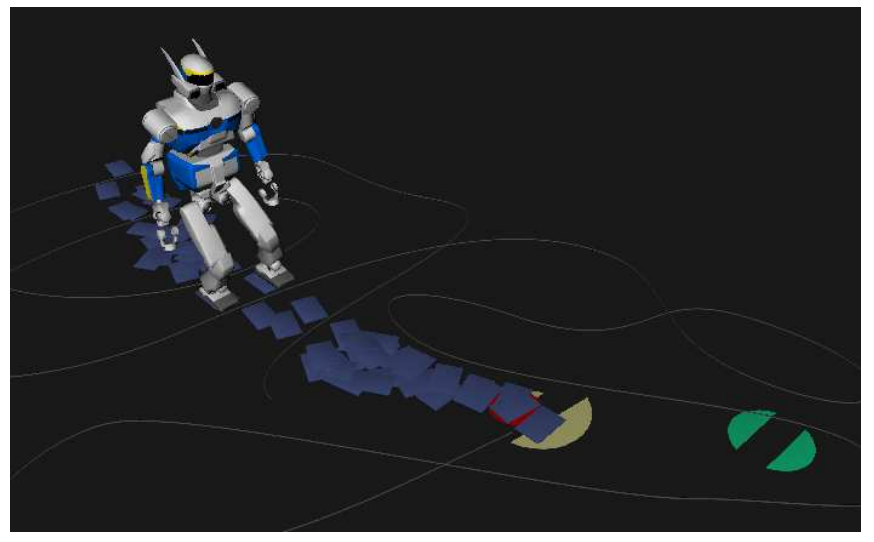

Fig. 9. The robot HRP-2 following a weakly collision-free path

"weak collision" checks, which are a bit more computationally costly, but can be handled efficiently with appropriate data structures, parallel or approximate approaches.

In the video accompanying this paper, we show a simple example on simulation: on a floor on which lies a long cable, the robot HRP-2 has to find a path until the green location (see Fig. 9). First we use PRM to plan the weakly collisionfree continuous motion of the $\Phi$ object, and then we generate footsteps that follow this path while avoiding the cable. Our implementation uses OMPL ([1]) for the PRM algorithm and PQP ([13]) for the collision checks.

Another class of techniques that can be easily applied are the nonholonomic motion planning algorithms, which for example aim at planning the motion of a car (see [14], [5], [10]). In our case, artificial constraints or control laws can be used to oblige the robot to execute maneuvers that look natural, or simply to favor forward motions rather than backward or lateral walking. To do motion planning with these control laws an algorithm such as KPIECE (for example) can be applied ([17]).

\section{GENERALIZATION TO DIFFERENT STEPPING CAPABILITIES}

The stepping capabilites considered so far are realistic, but the condition 6) in definition 2.2 might seem a bit restrictive. Here we consider the stepping capabilites with this constraint removed: in other words, a step is feasible as long as it involves two acceptable stances. Actually, we make another very slight change: the sets $\mathcal{E}_{\mathcal{L}}$ and $\mathcal{E}_{\mathcal{R}}$ are replaced by their interior. Thus, the definition of feasibility becomes:

Definition 6.1: The step defined by the 3 configurations $\left(\left(x_{L}, y_{L}, \theta_{L}\right),\left(x_{R}^{i}, y_{R}^{i}, \theta_{R}^{i}\right),\left(x_{R}^{f}, y_{R}^{f}, \theta_{R}^{f}\right)\right)$ is feasible if and only if the following constraints are verified:

1) $\left(x_{R}^{i}, y_{R}^{i}\right) \in \mathcal{E}_{\mathcal{R}}^{\circ}\left(x_{L}, y_{L}, \theta_{L}\right)$

2) $\left(x_{L}, y_{L}\right) \in \mathcal{E}_{\mathcal{L}}^{\circ}\left(x_{R}^{i}, y_{R}^{i}, \theta_{R}^{i}\right)$

3) $\left(x_{R}^{f}, y_{R}^{f}\right) \in \mathcal{E}_{\mathcal{R}}^{\circ}\left(x_{L}, y_{L}, \theta_{L}\right)$

4) $\left(x_{L}, y_{L}\right) \in \mathcal{E}_{\mathcal{L}}^{\circ}\left(x_{R}^{f}, y_{R}^{f}, \theta_{R}^{f}\right)$

5) Possibly additional constraints verifying the $\mu$-property (Property 2.1).

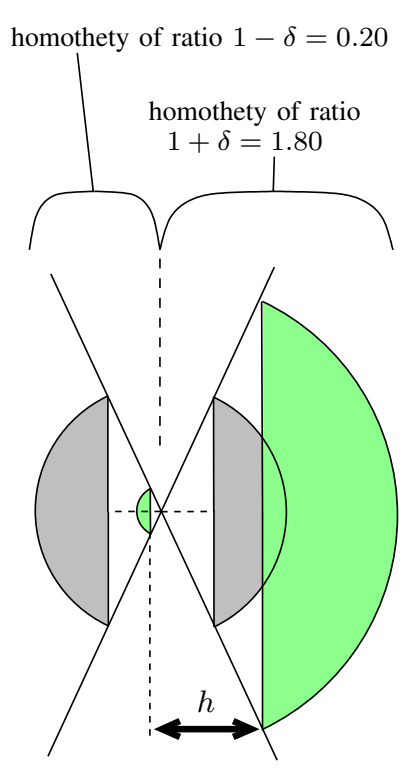

$\delta=0.80$

' homothety of ratio $1+\delta=0.85$

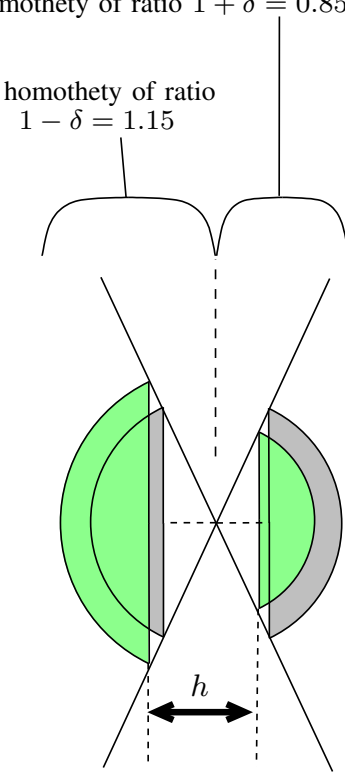

$\delta=-0.15$

Fig. 10. A new operation on $\Phi$ : if the first parameters of its configuration are $x, y$, and $\theta$, then for $\delta \in(-1,1), \Phi_{L}$ undergoes an homothety of center $(x, y)$ and ratio $1-\delta$, while $\Phi_{R}$ undergoes an homothety of center $(x, y)$ and ratio $1+\delta$ (thus $\Phi$ is unchanged if $\delta=0$ ).

An equivalence similar to theorem 2.1 can be obtained, but an additional operation on $\Phi$ is required. This operation depends on one parameter $\delta \in(-1,1)$, and is described on Fig. 10. It gives a fourth dimension to the configuration space of $\Phi$, which now becomes $\mathbb{R}^{2} \times S O(2) \times(-1,1)$. And the theorem becomes:

Theorem 6.1: There exists a collision-free sequence of feasible steps from $\left(\left(x_{L}^{0}, y_{L}^{0}, \theta_{L}^{0}\right),\left(x_{R}^{0}, y_{R}^{0}, \theta_{R}^{0}\right)\right)$ to the goal if and only if there exists a continuous weakly collision-free path $\mathcal{S}:[0,1] \mapsto \mathbb{R}^{2} \times S O(2) \times(-1,1)$ such that:

1) $\left(x_{L}^{0}, y_{L}^{0}\right) \in \Phi_{L}(\mathcal{S}(0))$ and $\left(x_{R}^{0}, y_{R}^{0}\right) \in \Phi_{R}(\mathcal{S}(0))$

2) $\left(x_{L}^{G}, y_{L}^{G}\right) \in \Phi_{L}(\mathcal{S}(1))$ and $\left(x_{R}^{G}, y_{R}^{G}\right) \in \Phi_{R}(\mathcal{S}(1))$

With a 4-dimensional configuration space, motion planning algorithms become a bit slower, but this theorem might be even more useful than the first one because with appropriate additional constraints the stepping capabilities obtained can be very close to the ones of real humanoid robots. To change the stepping capabilities even more, it is also possible to change the shape of $\mathcal{E}_{L}, \mathcal{E}_{R}$, and $\Phi$, or even the notion of weak collision. For example, let us consider the following notion of weak collision (with an adequate value for the new constant $d_{\text {min }}$ ):

Definition 6.2: A configuration $\Phi(x, y, \theta)$ of $\Phi$ is said to be "weakly collision-free" if and only if there exist $\left(x_{l}, y_{l}\right) \in$ $\Phi_{L}(x, y, \theta)$ and $\left(x_{r}, y_{r}\right) \in \Phi_{R}(x, y, \theta)$ such that there is no intersection between the obstacles and the two disks of radius $\frac{d_{F}}{2}$ and centers $\left(x_{l}, y_{l}\right)$ and $\left(x_{l}, y_{l}\right)$, and the distance between $\left(x_{l}, y_{l}\right)$ and $\left(x_{r}, y_{r}\right)$ is at least $d_{\text {min }}$. 


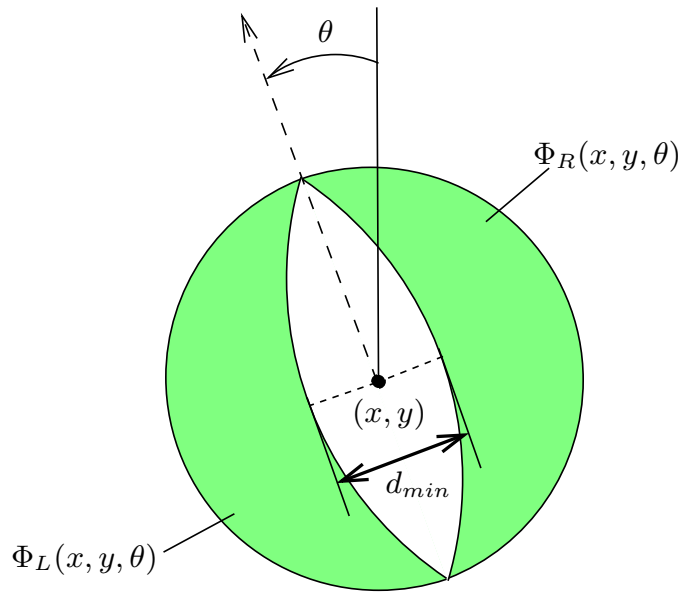

Fig. 11. An example of new $\Phi$ object that can be used when the notion of weak collision is slightly modified as in definition 6.2. This kind of object can be used to represent stepping capabilities that are very good approximations of the actual stepping capabilities of some humanoid robots, such as for instance HRP-2.

With this new notion of weak collision (which complexifies a bit the weak collision checks), theorems 2.1 and 6.1 can stay true with stepping capabilities and $\Phi$ objects that couldn't have been used so far, such as for example $\Phi$ objects with non-empty intersection between $\Phi_{L}$ and $\Phi_{R}$, which allow the robot to walk with its feet on a line (see fig. 11). General criteria for the acceptable shapes of $\Phi$ can be obtained, but are out of the scope of this paper.

Finally, by using 3D shapes that are somehow similar to the $2 \mathrm{D}$ shapes used in this paper, the theorems obtained can be extended to the case where relatively small $3 \mathrm{D}$ obstacles are taken into account.

\section{CONCLUSION AND FUTURE WORK}

In this paper, we showed an equivalence between $2 \mathrm{D}$ footstep planning and continuous planning for a $2 \mathrm{D}$ shape that can rotate and translate. We considered specific stepping capabilities but showed that a similar result can be obtain for variations of these stepping capabilities. Thanks to this result, a lot of classical and efficient algorithms for motion planning can be easily applied to footsep planning. In future work, we will try to use this equivalence as the basis of an algorithm for footstep planning with $3 \mathrm{D}$ obstacles, and apply it to a real robot.

More precisely, we will improve the bounding box method by using a bounding box that is divided into two parts and whose configuration depends on $x, y, \theta$ and additional parameters modifying the box geometry. On the upper part of the box classical collision checks will be performed, while on the lower part the notion of weak collision will be used instead. This way we will be able to keep the convenience and efficiency of the bounding box method without losing the robot ability to step over obstacles.

\section{ACKNOWLEDGMENTS}

This work was supported by a grant from the RBLINK Project, Contract ANR-08-JCJC-0075-01.

\section{REFERENCES}

[1] The Open Motion Planning Library (OMPL). http://ompl. kavrakilab.org, 2010.

[2] R. Alami, J.-P. Laumond, and Siméon T. Two manipulation planning algorithms. 1st Workshop on the Algorithmic Foundations of Robotics (WAFR'94), 1994.

[3] H. Arai. Motion planning of discrete-time nonholonomic systems. In 9th Int. Conf. on Advanced Robotics (ICAR'99), pages 577-583.

[4] Y. Ayaz, K. Munawar, M. Bilal Malik, A. Konno, and M. Uchiyama. Human-like approach to footstep planning among obstacles for humanoid robots. In IEEE/RSJ Int. Conf. on Intelligent Robots and Systems (IROS'06), 2006.

[5] J. Barraquand and J.-C. Latombe. Nonholonomic multibody mobile robots: Controllability and motion planning in the presence of obstacles. Algorithmica, 10:121-155, 1993.

[6] J.-M. Bourgeot, N. Cislo, and B. Espiau. Path-planning and tracking in a $3 \mathrm{~d}$ complex environment for an anthropomorphic biped robot. In IEEE Intl. Conf. on Intelligent Robots and Systems, volume 3, pages 2509-2514, 2002.

[7] J. Chestnutt, J. Kuffner, K. Nishiwaki, and S. Kagami. Planning biped navigation strategies in complex environments. In IEEE Int. Conf. on Humanoid Robotics (Humanoids'03), 2003.

[8] J. Chestnutt and J.J. Kuffner. A tiered planning strategy for biped navigation. In IEEE/RAS Int. Conf. on Humanoid Robotics (Humanoids'04), 2004.

[9] J. Chestnutt, M. Lau, G. Cheung, J. Kuffner, J. Hodgins, and T. Kanade. Footstep planning for the honda asimo humanoid. In IEEE Int. Conf. on Robotics and Automation (ICRA'05), pages 631-636.

[10] O. Lefebvre F. Lamiraux, D. Bonnafous. Reactive path deformation for nonholonomic mobile robots. IEEE Transactions on Robotics, 20(6):967-977, 2004.

[11] R. Geraerts and M.H. Overmars. A comparative study of probabilistic roadmap planners. In 5th Workshop on the Algorithmic Foundations of Robotics (WAFR'02), 2002.

[12] J. Kuffner, K. Nishiwaki, S. Kagami, M. Inaba, and H. Inoue. Footstep planning among obstacles for biped robots. In IEEE/RSJ Int. Conf. on Intelligent Robots and Systems (IROS'01), pages 500-505, 2001.

[13] E. Larsen, S. Gottschalk, M.C. Lin, and D. Manocha. Fast proximity queries with swept sphere volumes. Technical report, Univ. N. Carolina Chapel Hill, 1999.

[14] J.-P. Laumond. Feasible trajectories for mobile robots with kinematic and environment constraints. In Int. Conf. on Intelligent Autonomous Systems, pages 346-354, 1986.

[15] S.M. LaValle and J.J. Kuffner. Rapidly-exploring random trees: Progress and prospects. In 4th Workshop on the Algorithmic Foundations of Robotics (WAFR'00), pages 293-308, 2000.

[16] J.-P. Laumond O. Kanoun, E. Yoshida. An optimization formulation for footsteps planning. In IEEE/RAS Int. Conf. on Humanoid Robotics (Humanoids'09), 2009.

[17] I.A. Sucan and L.E. Kavraki. Kinodynamic motion planning by interior-exterior cell exploration. In 8th Workshop on the Algorithmic Foundations of Robotics (WAFR'08), 2008.

[18] E. Yoshida, C. Esteves, I. Belousov, J.-P. Laumond, T. Sakaguchi, and K. Yokoi. Planning 3D collision-free dynamic robotic motion through iterative reshaping. IEEE Trans. on Robotics, 24(5):1186-1198, 2008. 